\title{
Design of a highly magnified directional acoustic source based on the resonant cavity of two-dimensional phononic crystals
}

\author{
Tsung-Tsong $\mathrm{Wu}^{\text {a) }}$ Chung-Hao Hsu, and Jia-Hong Sun \\ Ultrasonics Laboratory, Institute of Applied Mechanics, National Taiwan University, Taipei 106, Taiwan
}

(Received 16 June 2006; accepted 14 September 2006; published online 26 October 2006)

\begin{abstract}
The authors report a design of a highly magnified directional acoustic source based on the planar resonant cavity of two-dimensional phononic crystals. The authors demonstrate that the order of the resonant mode and the reflective ratio of the double phononic crystal slab are the key factors to the magnified ratio of the directional acoustic amplifier. With properly designed mode and cavity width of the asymmetric phononic structure, the optimal magnified amplitude can be achieved by more than 86.5 times in comparison with the amplitude of the original line source freely radiating in water. (c) 2006 American Institute of Physics. [DOI: 10.1063/1.2370382]
\end{abstract}

Recently, there has been a growing concern for phononic crystals that demonstrate the band gaps of acoustic wave propagation in periodic elastic materials. The band gap phenomenon occurs to prohibit elastic waves of specific frequencies from traveling through the phononic crystals. ${ }^{1-3}$ In addition to the bulk acoustic waves, band gaps for surface acoustic waves have also been demonstrated. ${ }^{4-7}$ Large acoustic band gaps occur by several phononic crystal examples made of solid or fluid constituents. ${ }^{8-12}$ It may lead to the development of a variety of acoustic devices, such as sound insulation wall, acoustic wave guides, etc. Recently, Qiu et al. ${ }^{13}$ proposed an asymmetric structure of the planar cavity composed of phononic crystals to act as a directional acoustic source. With such a resonant cavity, acoustic waves can propagate a long distance with little spreading; however, the amplitude of the acoustic wave is only slightly increased.

In this letter, based on the resonant cavity proposed in Ref. 13, we propose a design of a highly magnified directional acoustic source. The proposed acoustic source not only preserves the same directional characteristics as that in Ref. 13; the amplitude of the radiative wave is also dramatically magnified. The calculations in our work are based on the finite difference time domain method. ${ }^{14}$ A parallelized computation program with a message passing interface ${ }^{15}$ is written and executed on a personal computer cluster system with eight processors.

The phononic crystal considered in this study is a solid/ fluid two-dimensional square lattice phononic crystal consisting of water as the base materials and steel as the cylinders. The lattice constant of the phononic crystal structure, hereunder referred to as $a$, is $10 \mathrm{~mm}$, and the radius of the cylinder is $3.75 \mathrm{~mm}$. In this case, the filling ratio $f$ is 0.442 . The density of steel is assumed as $7780 \mathrm{~kg} / \mathrm{m}^{3}$, and that of water is $1000 \mathrm{~kg} / \mathrm{m}^{3}$. The velocities of the longitudinal and transverse waves $C_{L}$ and $C_{T}$ in steel are 5825.22 and $3226.66 \mathrm{~m} / \mathrm{s}$, respectively, while the velocity of longitudinal waves in water is $1490 \mathrm{~m} / \mathrm{s}$.

A schematic diagram of the resonant cavity is demonstrated in Fig. 1. An asymmetric structure composed of six layers of crystals on the left and four layers of crystals on the right is designed to minimize the radiation toward the left. The width of the planar cavity is equal to $1.25 a$. The length of the resonant cavity along the $Y$ direction is $26 a$, and four layers of phononic structures are employed at both ends to suppress radiation along the $Y$ direction. Figure 2 shows the transmission coefficient and dispersion relation of the steel/ water phononic crystal. To calculate the transmission coefficients, a wideband wave packet is generated and propagates through the phononic crystal structure, and the spectrum is calculated and compared to that of free space. The solid line in the left of Fig. 2 is the result of the resonant cavity. Resonant modes of the cavity are excited, and sensors receive the resonant signals. Thus there are two resonant peaks, 58.52 and $86.46 \mathrm{kHz}$, located within the band gap in the $\Gamma X$ direction. The lower frequency peak appears in the partial band gap, and the higher frequency peak is in the complete band gap.

Shown in Fig. 3 is the near-field amplitude distribution with a line source of $86.46 \mathrm{kHz}$ (the resonant peak 2 in Fig. 2) located in the cavity. The darker color presents a larger amplitude value. As pointed out in Ref. 13, since the resonant frequency is in the complete band gap, the radiative waves from the source is directive (positive $X$ direction) and the spreading angle is very small. Observing the amplitude distribution along the centerline $\overline{A A^{\prime}}$, the amplitude of the directive waves is only about 1.3 times of the reference source amplitude. In the calculation, an alternative case as

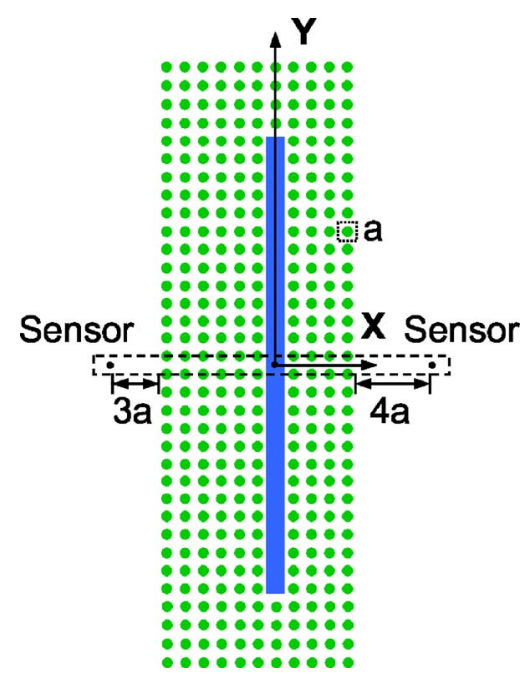

FIG. 1. (Color online) Schematics of the planar cavity. 


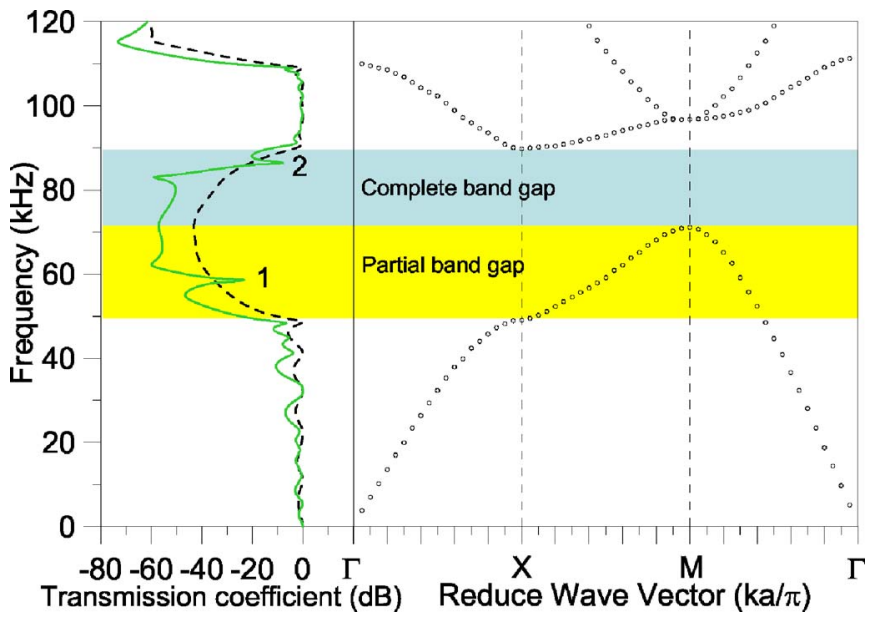

FIG. 2. (Color online) Dotted line in the left block represents the transmission coefficients for six layers of a phononic crystal slab in the $\Gamma X$ direction. The other line in the same block is the transmission coefficients of the resonant cavity in the $\Gamma X$ direction. On the right side of the figure is the dispersion relation of a steel/water square lattice phononic crystal with a filling ratio of 0.442 .

the rectangular dotted region shown in Fig. 1 with periodic conditions along the $Y$ direction is adopted and compared to the result of the full field case. Since the aperture of the cavity is large (26a) in comparison with the lattice constant, the results agree with each other well. Thus we utilize the periodic boundary conditions in the following studies and designs of the resonant cavity.

On the other hand, we note that the Fabry-Perot-type standing wave resonant condition indicates

$$
n \frac{\lambda_{n}}{2}=L_{n},
$$

where $n$ is the order of the resonant modes, $\lambda_{n}$ is the longitudinal wavelength, while $L_{n}$ is the equivalent cavity width for the $n$th order resonant mode. ${ }^{16}$ Since the boundaries of the resonant cavity comprising two phononic slabs are not purely fixed-end boundary conditions, the equivalent width of the cavity is expected to be different for different source frequencies. For the case of the second resonant frequency $(86.46 \mathrm{kHz})$ in Fig. 3 , by setting $n=2$ in Eq. (1) and the wave velocity of water as $1490 \mathrm{~m} / \mathrm{s}$, we find that the equivalent cavity width is $1.72 a$. A close look at Fig. 3 shows that the position of $X=0$ is an antinode (i.e., the amplitude is vanish-

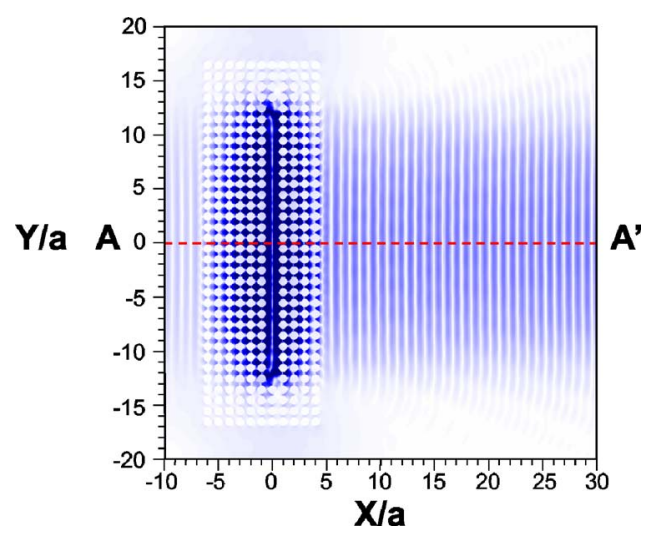

FIG. 3. (Color online) Near-field amplitude distribution with a line source set in a $1.25 a$ wide cavity; the operating frequency is $86.46 \mathrm{kHz}$. set in a $1.25 a$ wide cavity; the operating frequency is $86.46 \mathrm{kHz}$. $1.25 a, 0.83 a$, and $0.66 a$.
Downloaded 22 Dec 2008 to 140.112 .113 .225 . Redistribution subject to AlP license or copy

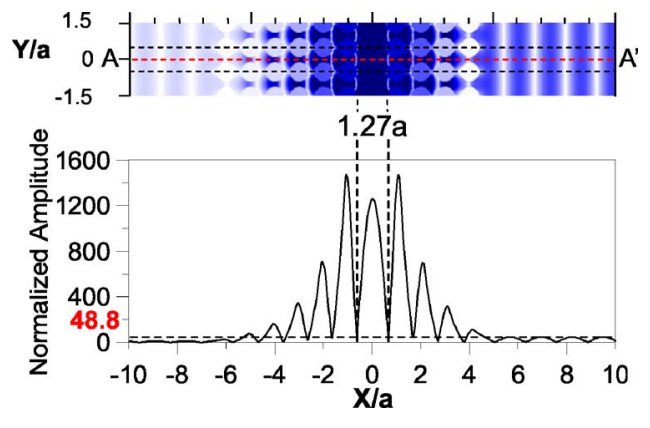

FIG. 4. (Color online) Wave amplitude distribution along the $X$ direction for the case of source frequency equal to $58.52 \mathrm{kHz}$, i.e., the first order resonance.

ing) and the distance between the first two adjacent antinodes is $1.72 a$, the same as that predicted by Eq. (1).

Figure 4 shows the wave amplitude distribution along the $X$ direction for the case of a $58.52 \mathrm{kHz}$ source frequency (the first resonant peak in Fig. 2). In this case, we find that the mode of resonance is $n=1$ and the wave amplitude in the right hand side is about 48.8 times of the reference source amplitude, while that at the left hand side is about 11.4 times. The equivalent cavity width measured from Fig. 4 is $1.27 a$ and is the same as that calculated from Eq. (1). It is noticeable that since this mode is not within the complete band gap, the radiative wave is not truly directional.

In the prior art, although the directivity is achieved in the design, the radiative amplitude remains unmagnified. To sum up, we observe that (1) if the resonant peak of the phononic resonant cavity is located in the complete band gap, high directivity of the radiative acoustic waves can be achieved and (2) utilization of the first order resonant mode leads to a magnified radiative wave amplitude. Therefore, to achieve an acoustic wave source that is not only highly directive but also highly magnified, it is necessary to adjust the width of the cavity and tune the resonant frequency of the first mode into the complete band gap.

Since the resonant frequency increases in proportion to the decrease of the cavity width, we decrease the cavity width and calculate the corresponding resonant spectrum to tune the first order resonance frequency into the complete band gap. Figure 5 shows the resonant spectra for cases of cavity widths equal to $1.25 a, 0.83 a$, and $0.66 a$. The case of $1.25 a$ is the same as that in Fig. 2, where the first resonant peak is located in the partial band gap and the second reso-

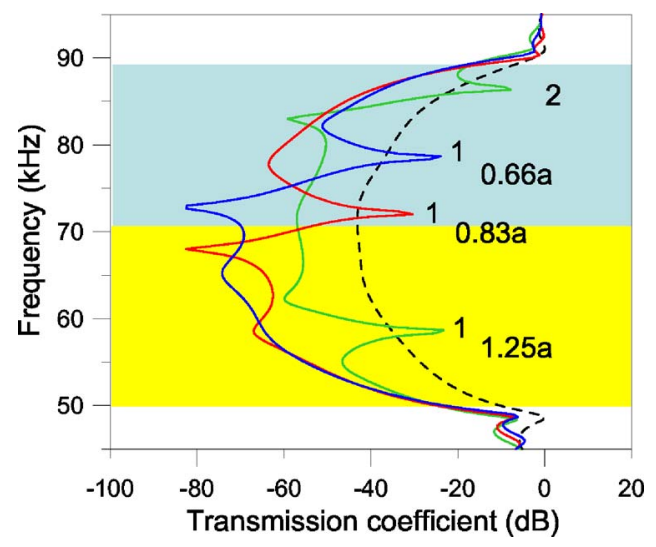

FIG. 5. (Color online) Resonant spectra for cases of cavity widths equal to

AIP license or copyright; see http://apl.aip.org/apl/copyright.jsp 


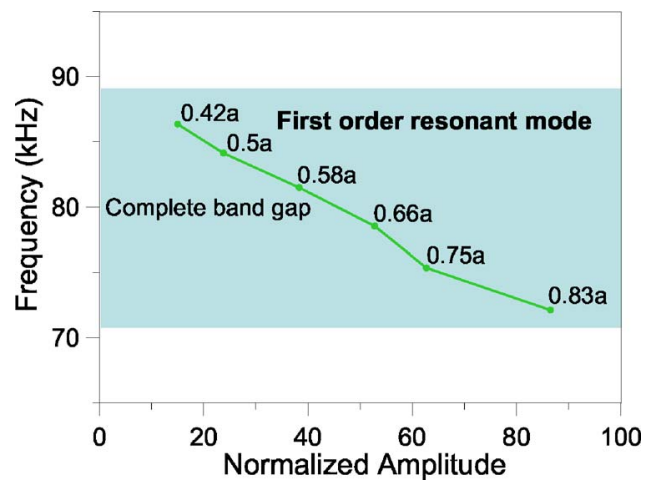

FIG. 6. (Color online) Magnification ratio vs resonant frequency of the first mode.

nant peak in the complete band gap. For the cases of $0.83 a$ and $0.66 a$, the first resonant peak is tuned into the complete band gap, while the second resonant peak is not in the complete band gap.

Shown in Fig. 6 is the magnification ratio versus the resonant frequency of the first mode. Six different cavity widths decreasing from $0.83 a$ to $0.42 a$ are studied. The results show that the magnification decreases, while the cavity width decreases. In other words, the optimal magnification can be achieved as the first order resonant frequency and is located slightly higher than the lower border of the complete band gap. A magnification of the amplitude of the radiated waves as high as 86.5 times has been achieved in this case. This phenomenon can be detected through the transmission coefficient of the six-layered phononic slab along the $X$ direction shown in the left of Fig. 2 (dotted line). On condition that the reflective coefficient around the lower border of the complete band gap is the biggest, whereas transmission coefficient is the smallest, the phononic resonant cavity can achieve the biggest resonance. While the cavity width becomes smaller (i.e., the first order resonance bounces higher) and the reflection coefficient decreases, the resonant amplitude would be decreased.
In conclusion, we report a design of a highly magnified directional acoustic source based on the planar resonant cavity of two-dimensional phononic crystals in this letter. The order of the cavity resonant mode and the reflection coefficient of the phononic crystal slab are the key factors in designing a directional acoustic amplifier. In the design, the first order resonant mode of the cavity is highly recommended for obtaining a much higher magnification ratio. To obtain a directional acoustic source, the first order resonant mode has to be tuned to the range of the complete band gap. Beyond that, the first resonant frequency is required to match with the highest reflection coefficient of the phononic crystal slab to obtain the highest magnification ratio. We note that the results of this study may find important applications in the design of a highly magnified acoustic source.

${ }^{1}$ M. S. Kushwaha, P. Halevi, L. Dobrzynski, and B. Djafari-Rouhani, Phys. Rev. Lett. 71, 2022 (1993).

${ }^{2}$ M. S. Kushwaha, P. Halevi, G. Martinez, L. Dobrzynski, and B. DjafariRouhani, Phys. Rev. B 49, 2313 (1994).

${ }^{3}$ M. S. Kushwaha and P. Halevi, Appl. Phys. Lett. 64, 1085 (1994).

${ }^{4}$ Y. Tanaka and S. I. Tamura, Phys. Rev. B 58, 7958 (1998).

${ }^{5}$ Y. Tanaka and S. I. Tamura, Phys. Rev. B 60, 13294 (1999).

${ }^{6}$ Tsung-Tsong Wu, Zi-Gui Huang, and S. Lin, Phys. Rev. B 69, 094301 (2004).

${ }^{7}$ Tsung-Tsong Wu, Liang-Chen Wu, and Zi-Gui Huang, J. Appl. Phys. 97, 094916 (2005).

${ }^{8}$ F. R. Montero de Espinosa, E. Jimenez, and M. Torres, Phys. Rev. Lett. 80, 1208 (1998).

${ }^{9}$ M. Torres, F. R. Montero de Espinosa, D. Garcia-Pablos, and N. Garcia, Phys. Rev. Lett. 82, 3054 (1999).

${ }^{10}$ M. Torres, F. R. Montero de Espinosa, and J. L. Aragon, Phys. Rev. Lett. 86, $4282(2001)$

${ }^{11}$ R. E. Vines, J. P. Wolfe, and A. V. Every, Phys. Rev. B 60, 11871 (1999).

${ }^{12}$ R. E. Vines and J. P. Wolfe, Physica B 263-264, 567 (1999).

${ }^{13}$ Chunyin Qiu, Zhengyou Liu, Jing Shi, and C. T. Chan, Appl. Phys. Lett. 86, 224105 (2005).

${ }^{14}$ Jia-Hong Sun and Tsung-Tsong Wu, Phys. Rev. B 71, 174303 (2005).

${ }^{15}$ Po-Feng Hsieh, Tsung-Tsong Wu, and Jia-Hong Sun, IEEE Trans. Ultrason. Ferroelectr. Freq. Control 53, 148 (2006).

${ }^{16}$ Chunyin Qiu, Zhengyou Liu, Jun Mei, and Jing Shi, Appl. Phys. Lett. 87, 104101 (2005). 